Special Issue of the 8th International Advances in Applied Physics and Materials Science Congress (APMAS 2018)

\title{
Effect of Thermo-Elastic Damping on Transverse Waves Propagating in a Single-Wall Carbon Nanotube
}

\author{
M.M. SELIM ${ }^{a, b, *}$ \\ ${ }^{a}$ Department of Mathematics, Al-Aflaj College of Science and Humanities Studies, \\ Prince Sattam bin Abdulaziz University, Al-Aflaj 710-11912 Saudi Arabia \\ ${ }^{b}$ Department of Mathematics, Suez Faculty of Science, Suez University, Egypt
}

\begin{abstract}
This paper concerned with the study of the effect of thermo-elastic damping on transverse waves propagating in a single-wall carbon nanotube. The $Q$-factor for thermo-elastic damping is investigated for transverse wave propagating in the nanotube. As a physical model, cylindrical shell is considered and the Donnell-MushtariVlasov approach is applied. The influences of the room temperature and radial thickness of the nanotube on the vibration behaviors are discussed. The effects of the room temperature and radial thickness of the nanotube on the quality factor are examined numerically. It can be shown that the $Q$-factor is proportional to the radial thickness of the nanotube. On the other hand, the opposite trend is appeared with the change of room temperature. It means that the $Q$-factor for a single-wall carbon nanotube decreases with the increase of the room temperatures.
\end{abstract}

DOI: 10.12693/APhysPolA.135.903

PACS/topics: transverse waves, thermo-elastic, damping, single-wall carbon nanotube, quality factor

\section{Introduction}

The discovery of carbon nanotubes (CNTs) has stimulated extensive research activities in science and engineering devoted to carbon nanostructures and nano composites. Many studies have shown that the carbon nanotubes possess remarkable mechanical and physical properties $[1,2]$ leading to many potential applications such as fluid transport, fluid storage at nano scale, and nano devices for drug delivery [3]. In addition to experimental endeavors, CNT modeling is classified into two main categories. The first is atomic modeling, which includes such techniques as classical molecular dynamics (MD) and tight-binding MD and the density functional model $[4,5]$. Li and Chou [6] reported an atomistic simulation of single-walled carbon nanotube (SWCNT) subjected to harmonic waves. Atomic modeling is limited to systems with a small number of molecules and atoms and is therefore confined to small-scale modeling. The second category is continuum modeling [7, 8], which includes classical (or local) beam and shell theories that are practical for analyzing CNTs for large-scale systems. Successful work has been conducted with continuum modeling, such as buckling analysis, dynamics studies, and mechanical property investigations of CNTs $[9,10]$. In various dissipation mechanisms, thermo-elastic damping has been identified as a dominant source of intrinsic dissipations in MEMS/NEMS oscillators [11]. Zener [12] has explored the results of thermo-elastic damping from the irreversible flow of heat driven by local temperature gradients that through the coupling accompany the strain field. Since the early work by Zener, quite a few

*e-mail: m.selim@psau.edu.sa studies relating to thermo-elastic vibration analysis for rod, beam, plate, and composite laminate structures have been reported [13-17].

Although the thermo-elastic damping in structures is not a new topic, the problems have been re-attracted increasing attentions more recently as the rapid development of micro- and nano-technology. However, only a limited portion of the literature is concerned with the vibration and buckling analysis of carbon nanotubes considering the thermal effects [18-20]. Zhang et al. [21] studied the thermal effect on the vibration of doublewalled carbon nanotubes using thermal elasticity. Wang et al. [22] studied the thermal effects on the vibration and instability of conveying fluid CNTs based on thermal elasticity mechanics. Hsu et al. [23] analyzed the frequency of chiral SWCNT subjected to thermal vibration and using Timoshenko beam model. Ni et al. [24] conducted an analysis of buckling behavior of single-walled CNTs subjected to axial compression under a thermal environment. Based on thermal elasticity mechanics, Zhang et al. [25] developed elastic multiple column model for column buckling of MWNTs with large aspect ratios under axial compression coupling with temperature change. They concluded that at low or room temperature the buckling strain including thermal effect is larger than that excluding the thermal effect and increases with the increase of temperature change. Alibeigloo et al. [26] investigated thermo-elastic analysis of functionally graded carbon nanotube-reinforced composite plate using theory of elasticity. Hoseinzadeh et al. [27, 28] have been studied the thermo-elastic vibration and damping analysis of double-walled carbon nanotubes based on shell theory.

In this paper, we derive an analytical expression for the natural frequency and the quality factor of the transverse waves propagating in a single-wall carbon nanotube due to thermo-elastic damping. The influences of the room 
temperature and radial thickness of the nanotube on the vibration behaviors are discussed. The effects of the room temperature and radial thickness of the nanotube on the $Q$-factor are examined numerically and the results are shown graphically.

\section{Formulation of the problem}

Assuming small strains and displacements, and considering the thin shell theory, Fig. 1 illustrates the cylindrical coordinate system $(x, \theta, z)$ and the geometry of the model. In the figure, $x, \theta$ and $z$-axis are longitudinal, circumferential and transverse directions, respectively. Furthermore, the dimensions of the model are presented in terms of the mean radius $R$, radial thickness $h$ and the mass density $\rho$.

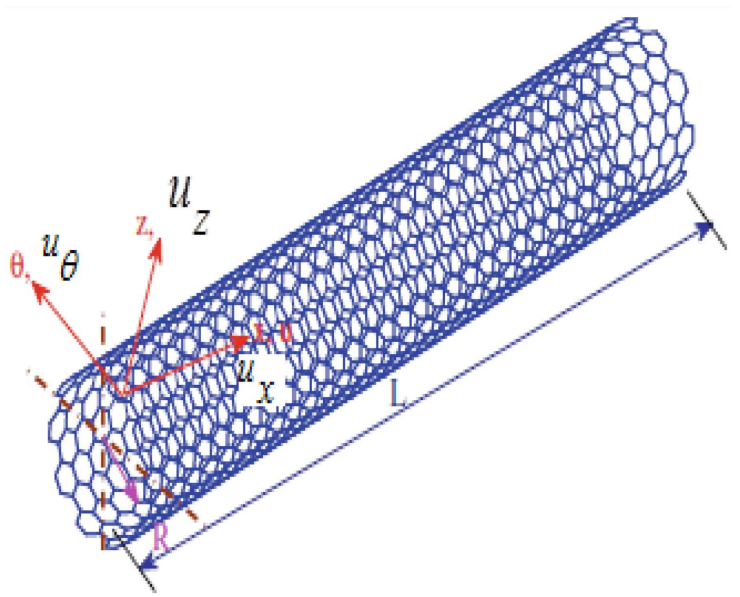

Fig. 1. Geometry of the tube with coordinate system .

The thermo-elastic linear equations of the cylindrical shell in $u_{x}, u_{\theta}$ and $u_{z}$ displacements can be written as [29]:

$$
\begin{aligned}
& \frac{\partial\left(N_{x}-N_{T}\right)}{\partial x}+\frac{1}{R} \frac{\partial N_{x \theta}}{\partial \theta}=\rho h \frac{\partial^{2} u_{x}}{\partial t^{2}}, \\
& \frac{\partial N_{x \theta}}{\partial x}+\frac{1}{R} \frac{\partial\left(N_{\theta}-N_{T}\right)}{\partial \theta}+\frac{1}{R} \frac{\partial M_{x \theta}}{\partial x} \\
& \quad+\frac{1}{R^{2}} \frac{\partial\left(M_{\theta}-M_{T}\right)}{\partial \theta}=\rho h \frac{\partial^{2} u_{\theta}}{\partial t^{2}}, \\
& \frac{\partial^{2}\left(M_{\theta}-M_{T}\right)}{\partial x^{2}}+\frac{2}{R} \frac{\partial^{2} M_{x \theta}}{\partial x \partial \theta}+\frac{1}{R^{2}} \frac{\partial^{2}\left(M_{\theta}-M_{T}\right)}{\partial \theta^{2}} \\
& -\frac{N_{\theta}-N_{T}}{R}=\rho h \frac{\partial^{2} u_{z}}{\partial t^{2}},
\end{aligned}
$$

And the strain-displacements relations are written as:

$$
\begin{aligned}
& \varepsilon_{x}=\varepsilon_{x}^{0}+z \kappa_{x}, \quad \varepsilon_{\theta}=\varepsilon_{\theta}^{0}+z \kappa_{\theta}, \\
& \varepsilon_{x \theta}=\varepsilon_{x \theta}^{0}+z \kappa_{x \theta},
\end{aligned}
$$

where $\varepsilon_{z}, \varepsilon_{\theta}$ and $\varepsilon_{x}$ are the transverse, circumferential and longitudinal components of strains, respectively.

$$
\begin{aligned}
& \varepsilon_{x}^{0}=\frac{\partial u_{x}}{\partial x}, \quad \varepsilon_{\theta}^{0}=\frac{1}{R}\left(\frac{\partial u_{\theta}}{\partial \theta}+u_{z}\right), \\
& \varepsilon_{x \theta}^{0}=\frac{\partial u_{\theta}}{\partial x}+\frac{1}{R} \frac{\partial u_{x}}{\partial \theta}
\end{aligned}
$$

and

$$
\begin{aligned}
& \kappa_{x}=-\frac{\partial^{2} u_{z}}{\partial x^{2}}, \quad \kappa_{\theta}=\frac{1}{R^{2}}\left(\frac{\partial u_{\theta}}{\partial \theta}-\frac{\partial^{2} u_{z}}{\partial \theta^{2}}\right), \\
& \kappa_{x \theta}=\frac{1}{R}\left(\frac{\partial u_{\theta}}{\partial x}-2 \frac{\partial^{2} u_{z}}{\partial x \partial \theta}\right),
\end{aligned}
$$

are the curvatures. Furthermore, the membrane forces and bending moments are defined as

$$
\begin{aligned}
& N_{x}=\Delta\left(\varepsilon_{x}^{0}+\Omega \varepsilon_{\theta}^{0}\right), \quad N_{\theta}=\Delta\left(\varepsilon_{\theta}^{0}+\Omega \varepsilon_{x}^{0}\right), \\
& N_{x \theta}=\frac{\Delta(1-\Omega) \varepsilon_{x \theta}^{0}}{2}, \\
& M_{x}=D\left(\kappa_{x}+\Omega \kappa_{\theta}\right), \quad M_{\theta}=D\left(\kappa_{\theta}+\Omega \kappa_{x}\right), \\
& M_{x \theta}=\frac{D(1-\Omega)}{2} \kappa_{x \theta},
\end{aligned}
$$

where $\left.\Delta=E h / 1-\Omega^{2}\right)$ is the membrane stiffness, $D=E h^{3} /\left[12\left(1-\Omega^{2}\right)\right]$ is the bending stiffness, $\Omega$ is the Poisson ratio and $E$ is the elasticity constant. Also, membrane force $N_{T}$ and bending moment $M_{T}$ due to thermal effect can be expressed as

$$
\begin{aligned}
& N_{T}=\frac{E \alpha_{t}}{1-\Omega} \int_{-h / 2}^{h / 2}\left(T-T_{0}\right) \mathrm{d} z, \\
& M_{T}=\frac{E \alpha_{t}}{1-\Omega} \int_{-h / 2}^{h / 2}\left(T-T_{0}\right) z \mathrm{~d} z,
\end{aligned}
$$

where $\alpha_{t}$ is the coefficient of thermal expansion.

The temperature distribution for thermal flow coupled with strain can be obtained from the heat conduction equation as [30]:

$$
\chi \hat{\nabla}^{2} T=\rho C_{p} \frac{\partial T}{\partial t}+\frac{E \alpha_{t} T}{(1-2 \Omega)} \frac{\partial e}{\partial t},
$$

where $\chi$ is the thermal conductivity, $C_{p}$ is the heat capacity coefficient at constant pressure, and $e$ is the dilatation strain due to the thermal effect, which define as

$$
e=\varepsilon_{x}+\varepsilon_{\theta}+\varepsilon_{z}
$$

For cylindrical thin shell problems, the normal stress, $\sigma_{z}$, along the thickness direction is neglected. The stress-strain relationships with thermal effects thus can be written as

$$
\begin{aligned}
& \varepsilon_{x}=\frac{1}{E}\left(\sigma_{x}-\Omega \sigma_{\theta}\right)+\alpha_{t}\left(T-T_{0}\right), \\
& \varepsilon_{\theta}=\frac{1}{E}\left(\sigma_{\theta}-\Omega \sigma_{x}\right)+\alpha_{t}\left(T-T_{0}\right), \\
& \varepsilon_{z}=-\frac{\Omega}{E}\left(\sigma_{\theta}+\sigma_{x}\right)+\alpha_{t}\left(T-T_{0}\right),
\end{aligned}
$$

Solving the above equation for $\varepsilon_{z}$ in terms of $\varepsilon_{x}$ and $\varepsilon_{\theta}$, we obtain 


$$
\varepsilon_{z}=\frac{1}{1-\Omega}\left[-\Omega\left(\varepsilon_{x}+\varepsilon_{\theta}\right)+(1+\Omega) \alpha_{t}\left(T-T_{0}\right)\right] .
$$

Based on well-known Donnell-Mushtari-Vlasov (DMV) approach [31], the governing equations can be simplified for transverse displacement-dominated vibrations. Then, the terms involving the in-plane displacements $\left(u_{x}, u_{\theta}\right)$ are neglected only in the bending strains based on this method. Thus, Eq. (6) and the first two equations in Eq. (1) in can be rewritten as

$$
\begin{aligned}
& \kappa_{x}=-\frac{\partial^{2} u_{z}}{\partial x^{2}}, \quad \kappa_{\theta}=-\frac{1}{R^{2}}\left(\frac{\partial^{2} u_{z}}{\partial \theta^{2}}\right), \\
& \kappa_{x \theta}=-\frac{2}{R}\left(\frac{\partial^{2} u_{z}}{\partial x \partial \theta}\right),
\end{aligned}
$$

With relations (7) and (14) and considering the geometric condition of the transverse wave propagating in single-walled carbon nanotubes as [32]:

$$
\frac{\partial}{\partial \theta}=0, \quad u_{x}=-z \frac{\partial u_{z}}{\partial x}
$$

The thermo-elastic linear equation of motion governing the transverse deflection of the single-walled nanotube becomes [33]:

$$
D \frac{\partial^{4} u_{z}}{\partial x^{4}}+\frac{\partial^{2} M_{T}}{\partial x^{2}}+\frac{\partial^{2} u_{z}}{\partial x^{2}} N_{T}+\rho h \frac{\partial^{2} u_{z}}{\partial t^{2}}=0 .
$$

Assuming small strains and negligible in-plane displacements, the strains are related to $u_{z}$ as [27]

$$
\varepsilon_{x}=-z \frac{\partial^{2} u_{z}}{\partial x^{2}}, \quad \varepsilon_{\theta}=-z \frac{\partial^{2} u_{z}}{\partial z^{2}} .
$$

Substituting Eqs. (13) and (17) into Eq. (11), we obtain

$$
e=-\left(\frac{1-2 \Omega}{1-\Omega}\right) z \nabla^{2} u_{z}+\frac{1+\Omega}{1-\Omega} \alpha_{t}\left(T-T_{0}\right)
$$

In most applications, $\mathrm{T}$ on the second term of the righthand side of Eq. (10) can be replaced by T0 to yield a linear equation for the temperature [28]. Therefore, the heat Eq. (10) can be rewritten as

$$
\begin{gathered}
\chi \hat{\nabla}^{2} T=\left(\rho C_{p}+\frac{E \alpha_{t}^{2} T_{0}(1+\Omega)}{(1-\Omega)(1-2 \Omega)}\right) \frac{\partial T}{\partial t} \\
-\frac{E \alpha_{t} T_{0}}{(1-\Omega)} \frac{\partial}{\partial t}\left(z \nabla^{2} u_{z}\right) .
\end{gathered}
$$

Typically, $\rho C_{p} \gg \frac{E \alpha_{t}^{2} T_{0}(1+\Omega)}{(1-\Omega)(1-2 \Omega)}[33]$. Hence, Eq. (19) reduces to

$$
\chi \hat{\nabla}^{2} T=\rho C_{p} \frac{\partial T}{\partial t}-\frac{E \alpha_{t} T_{0}}{(1-\Omega)} \frac{\partial}{\partial t}\left(z \nabla^{2} u_{z}\right) .
$$

The thermal gradient $\hat{\nabla}^{2} T$ for cylindrical shell is

$$
\hat{\nabla}^{2} T=\frac{1}{R+z} \frac{\partial T}{\partial z}+\frac{\partial^{2} T}{\partial z^{2}}+\frac{1}{(R+z)^{2}} \frac{\partial^{2} T}{\partial \theta^{2}}+\frac{\partial^{2} T}{\partial x^{2}}
$$

Further, Eq. (21) can be reduced by considering the first terms on right-hand side only because thermal gradients in the thickness direction $\mathrm{z}$ are much larger than the other directions [34].

$$
\chi \frac{\partial^{2} T}{\partial z^{2}}=\rho C_{p} \frac{\partial T}{\partial t}-\frac{E \alpha_{t} T_{0}}{(1-\Omega)} \frac{\partial}{\partial t}\left(z \nabla^{2} u_{z}\right) .
$$

Equations (16) and (22) are the simplified thermoelastic equation under transverse deflection-dominated vibrations, and can be used for the analysis of thermoelastic damping of transverse waves propagating in the single-wall carbon nanotube.

\section{Harmonic solution of transverse waves}

For propagation of transverse waves in any arbitrary direction, the solution of Eqs. (16) and (22) can be expressed as:

$$
\begin{aligned}
& u_{z}(x, z, t)=\psi(x, z) \mathrm{e}^{\mathrm{i} \omega t}, \\
& T(x, \theta, z, t)-T_{0}=\Theta(x, \theta, z) \mathrm{e}^{\mathrm{i} \omega t},
\end{aligned}
$$

where $\omega$ is the circular frequency and $i=\sqrt{-1}$.

Substituting Eqs. (23) into Eqs. (16) and (22), and noting that the temperature variation across the nanotube thickness is much larger than its variation across the plane of the nanotube, we obtain

$$
\begin{gathered}
D\left(\frac{\partial^{4} \psi}{\partial x^{4}}\right)+\frac{E \alpha_{t}}{(1-\Omega)} \int_{-h / 2}^{h / 2} z \frac{\partial^{2} \Theta}{\partial x^{2}} \mathrm{~d} z \\
+\frac{E \alpha_{t}}{(1-\Omega)} \frac{\partial^{2} \psi}{\partial x^{2}} \int_{-h / 2}^{h / 2} \Theta \mathrm{d} z=\rho h \omega^{2} \psi, \\
\frac{\partial^{2} \Theta}{\partial z^{2}}-\lambda^{2} \Theta=-\frac{\mathrm{i} E \alpha_{t} T_{0} \omega}{\chi(1-\Omega)} z \frac{\partial^{2} \psi}{\partial x^{2}},
\end{gathered}
$$

where $\lambda=\sqrt{\frac{i \omega \rho C_{p}}{\chi}}$.

The heat flux between the surfaces and surroundings of the nanotube is sufficiently small; so, zero heat flux conditions can be applied.

$$
\frac{\partial \Theta}{\partial z}=0 \quad \text { at } \quad z= \pm \frac{h}{2}
$$

Finally, the temperature profile coupled with the displacement and stress function can be obtained as

$$
\Theta=\frac{E \alpha_{t} T_{0}}{\rho C_{p}(1-\Omega)} \frac{\partial^{2} \psi}{\partial x^{2}}\left(z-\frac{\sin (\Pi z)}{\Pi \cos \left(\frac{\Pi h}{2}\right)}\right),
$$

where $\Pi=(1-\mathrm{i}) \sqrt{\frac{\omega \rho C_{p}}{2 \chi}}$.

Substituting Eq. (27) into Eq. (24) and carrying out the integrations and retaining the linear terms, we obtain

$$
\begin{aligned}
& {\left[D+\frac{E^{2} \alpha_{t}^{2} T_{0}}{\rho C_{p}(1-\Omega)^{2}}\left(\frac{h^{3}}{12}+\frac{h}{\Pi^{2}}-\frac{2 \tan (\Pi h / 2)}{\Pi^{3}}\right)\right]} \\
& \quad \times \frac{\partial^{4} \psi}{\partial x^{4}}=\rho h \omega^{2} \psi .
\end{aligned}
$$

Then, appropriate assumed mode shapes are chosen in order to obtain the natural frequency under thermo-elastic damping. For vibration of single-walled carbon nanotubes with two ends simply-supported, the solution has the form: 


$$
\psi(x, z)=\zeta \sin \left(\frac{m \pi x}{L}\right) \cos (n z),
$$

where $\zeta$ is the real number denotes the radial displacement amplitude, $m$ is the axial half wave number, and $n$ is the circumferential wave number.

Substituting Eq. (29) into Eq. (28), we obtain

$$
\begin{aligned}
\omega^{2} & =\left(\frac{m \pi x}{L}\right)^{4} \\
& \times\left[D+\frac{E^{2} \alpha_{t}^{2} T_{0}}{\rho C_{p}(1-\Omega)^{2}}\left(\frac{h^{3}}{12}+\frac{h}{\Pi^{2}}-\frac{2 \tan (\Pi h / 2)}{\Pi^{3}}\right)\right] .
\end{aligned}
$$

Lastly, analytical expression of the quality factor $(Q$ factor) of the single-wall carbon nanotube in this case take the form:

$$
Q=\left|\frac{D}{2 \operatorname{Im} \delta(\omega)}\right|,
$$

where $\delta(\omega)=\frac{E^{2} \alpha_{t}^{2} T_{0}}{\rho C_{p}(1-\Omega)^{2}}\left(\frac{h^{3}}{12}+\frac{h}{\Pi^{2}}-\frac{2 \tan (\Pi h / 2)}{\Pi^{3}}\right)$.

\section{Numerical results}

In this section, verification of this work is presented by considering the effect of thermo-elastic damping on transverse waves propagating in single-wall carbon nanotube (SWCN) with an, which has been analytically examined by applying heat conduction equation of the shell. Mechanical and thermal properties are listed below.

The material constants are the Young modulus $E=$ $1060 \mathrm{GPa}$, the mass density $\rho=2270 \mathrm{~kg} \mathrm{~m}^{-3}$, the Poisson ratio $\Omega=0.25$, heat capacity coefficient $\rho C_{p}=1.36 \times 10^{6} \mathrm{Jm}^{3} \mathrm{~K}^{-1}$, thermal expansion coefficient $\alpha_{t}=7 \times 10^{-6} \mathrm{~K}^{-1}$, thermal conductivity $\chi=$ $2000 \mathrm{Js}^{-1} \mathrm{~m}^{-1} \mathrm{~K}^{-1}$ [40].

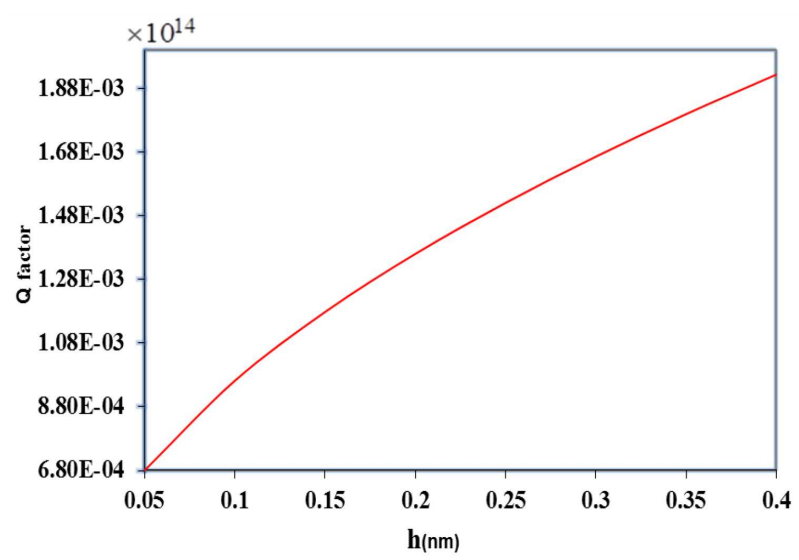

Fig. 2. Variation of the $Q$-factor with the radial thickness $(h)$ of SWCN for the transverse wave propagation.

At first, the effect of the temperature changes and radial thickness of a single-walled carbon nanotube on the $Q$-factor is investigated. Secondly, the variation of thermo-elastic damping with $T_{0}$ and $h$ are plotted in Figs. 2 and 3. The Variations of the $Q$-factor with the radial thickness $(h)$ of SWCN are shown in Fig. 2. Meanwhile, the results for thermo-elastic damping with differ-

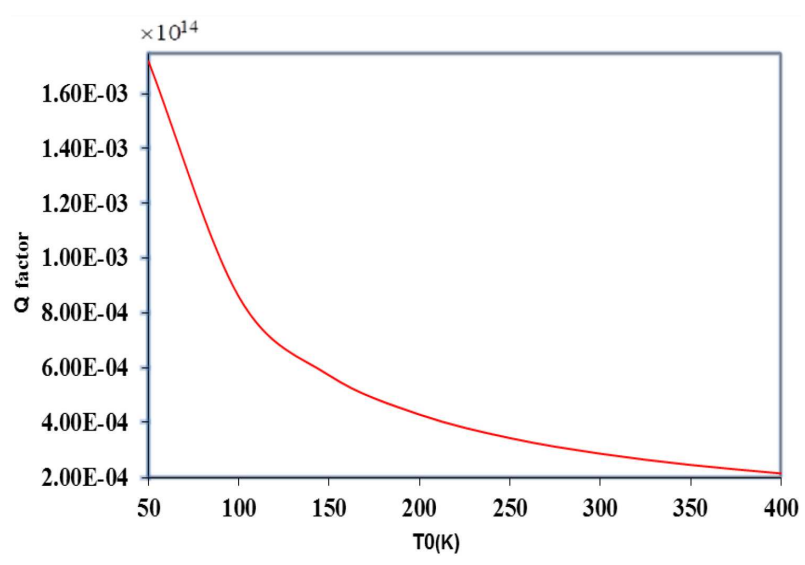

Fig. 3. Variation of the $Q$-factor with the temperature $T_{0}$ for the transverse waves propagation.

ent temperature changes $(T=50 \mathrm{~K}$ to $400 \mathrm{~K})$ are shown in Fig. 3. In the Fig. 2, the $Q$-factor of the nanotube is shown to be proportional to the radial thickness of SWCN.

It is can see that, the quality factor of the nanotube increases as the radial thickness increases. On the other hand, Fig. 3 displays the opposite trend. The quality factor decreases as the room temperature increases. These results can be helpful in the design of resonators.

\section{Conclusion}

In this research paper, the quality factor for thermoelastic damping is investigated for transverse wave propagating in a single wall carbon nanotube ( $\mathrm{SWCN})$. As a physical model, cylindrical shell is considered. In order to simplify the equations of motion, DMV approach is applied. By introducing the stress function, the compatibility and heat conduction equations can be solved with reference to a simplified equation of motion. The $Q$-factor are determined analytically. The influences of the room or low temperature and radial thickness of the nanotube on the vibration behaviors are discussed, respectively. Then, the effects of the room temperature and radial thickness of the nanotube on the $Q$-factor are closely examined numerically. It can be shown that the $Q$-factor is proportional to the radial thickness of the nanotube. On the other hand, the increase of the radial thickness of the nanotube leads to an increase in thermoelastic damping. It can be also seen that the $Q$-factor for a SWCN decreases with the increase of the room temperatures.

\section{Acknowledgments}

The authors wish to express gratitude for the supports provided by the King Abdulaziz City for Science and Technology (KACST), Award Number (SGP-35-7) for this research work. The author also would like to acknowledge the supports provided by the Deanship of Scientific Research of prince Sattam Bin Abdulaziz University during this research work. 


\section{References}

[1] E.T. Thostenson, Z. Ren, T.W. Chou, Rev. Comp. Sci. Tech. 61, 1899 (2001).

[2] L.S. Schadler, S.C Giannaris, P.M Ajayan, Appl. Phys. Lett. 73, 3842 (1998).

[3] A.B. Dalton, S. Collins, J. Razal, E. Munoz, V.H. Ebron, B.G. Kim, J. Mater. Chem. 14, 1 (2004).

[4] S. Iijima, Nature 354, 56 (1991).

[5] P. Ball, Nature 414, 142 (2001).

[6] D. Sanchez-Portal, E. Artacho, J.M. Soler, A. Rubio, P. Ordejon, Phys. Rev. B 59, 12678 (1999).

[7] B.I. Yakobson, M.P. Campbell, C.J. Brabec, J. Bernholc, Comput. Mater. Sci. 8, 341 (1997).

[8] K.M. Liew, X.Q. He, C.H. Wong, Acta Mater. 52, 2521 (2004)

[9] X. Yao, Q. Han, Eur. J. Mech. A 26, 298 (2007).

[10] C.Q. Ru, Phys. Rev. B 62, 10405 (2000)

[11] A.H. Nayfeh, M.I. Younis, J. Micromech. Microeng. 14, 1711 (2004).

[12] C. Zener, Elasticity and anelasticity of metals, University of Chicago Press, Chicago 1948

[13] A.S. Nowick, B.S. Berry, Anelastic relaxation in crystalline solids, Academic Press, New York 1972.

[14] P. Chadwick, J. Mech. Phys. Solids 10, 99 (1962).

[15] R.C. Shieh, J. Appl. Mech. 42, 405 (1975).

[16] B.H. Houston, D.M. Photiadis, M.H. Marcus, J.A. Bucaro, X. Liu, Appl. Phys. Lett. 80, 1300 (2002).

[17] S. Sapmaz, Y.M. Blanter, L. Gurevich, H.S.J. van der Zant, Phys. Rev. B 67, 235414 (2003).
[18] V. Vasu, T. Abhinav, J. Mech. Eng. 1, 1 (2011).

[19] F.L. Guo, J. Therm. Stress. 36, 1156 (2013).

[20] M.V. Ferreira, M. Rivera, J. Angew, Math. Phys. 69, 1751 (2018)

[21] Y.Q. Zhang, X. Liu, G.R. Liu, Nanotech. 18, 445701 (2007).

[22] L. Wang, Q. Ni, M. Li, Q. Qian, Phys. E 40, 3179 (2008).

[23] J.C. Hsu, R.P. Chang, W.J. Chang, Phys. Lett. A 372, 2757 (2008).

[24] B. Ni, S.B. Sinnott, P.T. Mikulski, J.A. Harrison, Phys. Rev. Lett. 88, 205505 (2002).

[25] Y.Q. Zhang, X. Liu, J.H. Zhao, Phys. Lett. A 372 , 1671 (2008)

[26] Alibeigloo, K.M. Liew, Composite Structures 106, 873 (2013).

[27] M.S. Hoseinzadeh, S.E. Khadem, Phys. E 43, 1146 (2011).

[28] M.S. Hoseinzadeh, S.E. Khadem, Phys. E 57, 6 (2014).

[29] W. Scedel, J. Sound. Vib. 70, 309 (1980).

[30] S.J. Wong, C.H.J. Fox, S. McWilliam, C.P. Fell, R. Eley, J. Micromech. Microeng. 14, S108 (2004).

[31] W. Scedel, Vibrations of Shells and Plates, Dekker, New York 2004.

[32] J.D. Achenbach, Wave Propagation in Elastic Solids, North-Holland, Amsterdam 1973.

[33] A.H. Nayfeh, M.I.Younis, J. Micromech. Microeng. 14, 1711 (2004).

[34] P. Lu, H.P. Lee, C. Lu, H.B. Chen, International J. Mech. Sci. 50, 501 (2008). 